\title{
Study of dust particle size distribution in the air of work areas at cement production facilities
}

\author{
Nadezhda Menzelintseva*, Natalia Karapuzova, Awadh M. Redhwan, Ekaterina Fomina \\ Volgograd State Technical University, 400301, Volgograd, Russia
}

\begin{abstract}
The particle size distribution of dust in the air of the work area has been determined for some cement plant shops. An experimental study has been conducted to explore the effects of microclimate parameters on the dust particle size distribution on the shop floor in the cement milling shop and cement packing shop, and regression equations have been obtained. A mathematical model has been developed to forecast the dust particle size distribution in the air of work areas of cement plants.
\end{abstract}

\section{Introduction}

The main sources of dust emission in the process shops of cement production facilities are mills, kilns, belt, scraper or tray conveyors transporting dusty materials, as well as cement screening and packing machines. The dust levels in various work areas of cement plants may be tenfold the rated parameters or even greater [1].

Inhalation of dust over extended periods of time may cause occupational diseases, among them: dust fibrosis (pneumoconiosis), dust bronchitis, conjunctivitis, various skin diseases, which is why it is critical to devise appropriate measures to reduce the dust content on the shop floor [2].

The leading causes of shop air contamination with dust are: insufficient equipment containment, incorrect sizing and design of local exhausts, low efficiency of dust aspiration systems, open transportation of dusty materials. The dust situation in the work area is largely determined by the microclimate parameters; however, there has been a lack of attention to the effects of temperature, humidity and mobility of the air in work area on the content of particulate matter in the cement production process [3].

\section{Objective and tasks of research}

To determine the particle size distribution of cement dust emitted by the Sebryakovcement plant in Mikhailovka, Volgograd Region, an experimental study was conducted focused on the distribution of dust particles in the air of the cement milling shops, near the mill feeder, in the rotary kiln operator's work area, and in the cement packing shop in the packing machine operator's work area. The results of the analysis of dust particle size distribution

\footnotetext{
*Corresponding author: karapuzova_ny@mail.ru
} 
are provided as integral functions of particle mass distribution according to particle diameters.

The particle size range of cement dust sampled next to the mill feeder in the cement milling shop is $(0.1-7) \mu \mathrm{m}, \mathrm{d}_{50}=4.3 \mu \mathrm{m}$. The content of $\mathrm{PM}_{2.5}$ particles is $10 \%$. The particle size of cement dust sampled near the mill in the cement milling shop fall within a range of $0-18 \mu \mathrm{m}, \mathrm{d}_{50}=10 \mu \mathrm{m}$. The share of $\mathrm{PM}_{10}$ particles accounts for $51 \%$, the share of $\mathrm{PM}_{2.5}$ particles - for $3.5 \%$.

The analysis of the data obtained showed that the particle size range of dust emitted into the air surrounding the kiln at the calcination stage is $(0.1-7) \mu \mathrm{m}$, the median size is $\mathrm{d}_{50}=4.5$ $\mu \mathrm{m}$. The share of $\mathrm{PM}_{2.5}$ particles accounts for $15 \%$ (fig. 1 ).

The analysis of particle size distribution of cement dust sampled in the packing worker's breathing area in the cement packing shop showed that the size of dust particles varies within a range of $1-12 \mu \mathrm{m}, \mathrm{d}_{50}=5.5 \mu \mathrm{m}$. The share of $\mathrm{PM}_{10}$ particles accounts for $99 \%$, the share of $\mathrm{PM}_{2.5}$ particles - for $7.5 \%$.

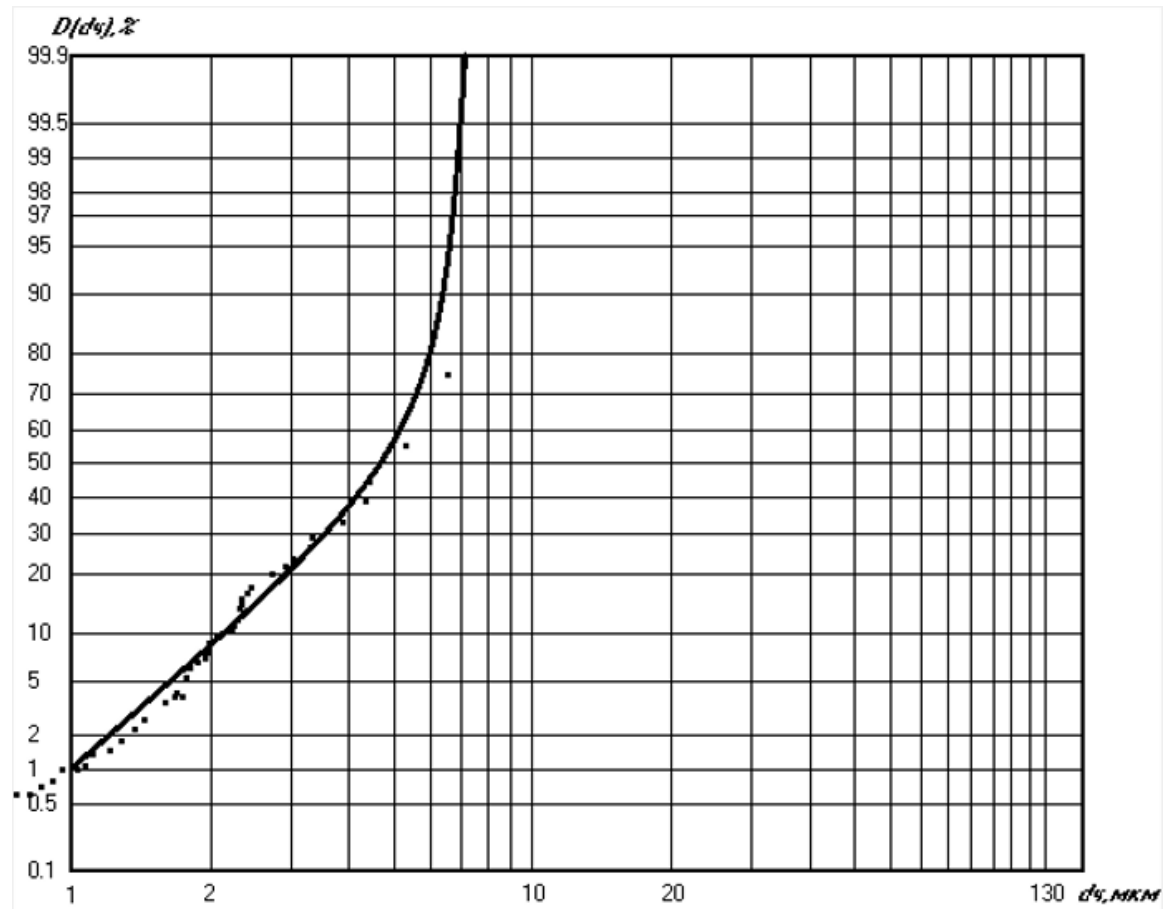

Fig. 1. Integral curve of mass-size distribution in the probabilistic logarithmic net for dust sampled near the kiln at the calcination stage: $\mathrm{PM}_{10}-0 \%, \mathrm{PM}_{2.5}-15 \%$

The development of the dust situation in the work area as well as the dust load on the respiratory organs of the workers are greatly influenced by the microclimate parameters.

In order to study the effects of the microclimate parameters on the dust particle size distribution in the air of the work area, an experiment was conducted according to plan $\mathrm{B}_{3}$ covering the cement milling shop and the cement packing shop [4]. The response surface functions were as follows: $\mathrm{Y}_{1}-$ content of $10 \mu \mathrm{m}$ particles $\mathrm{D}(\mathrm{PM} 10), \mathrm{Y}_{2}-$ content of $2.5 \mu \mathrm{m}$ particles $\mathrm{D}(\mathrm{PM} 2,5), \mathrm{y}_{3}-$ content of median-size particles $\mathrm{D}(50)$. The variable factors were assumed to be: $X_{1}-$ air humidity $(\varphi), X_{2}$-air temperature $(t), X_{3}$-air mobility in the work area $(\mathrm{V})$.

Adequate regression equations were obtained, which, given only the relevant regression coefficients in the denominated quantities, are expressed as shown below: 
The relevance of the equation coefficients was verified using Student's t-test, and the adequacy was verified using Fisher's criterion [4].

for the cement milling shop:

$$
\begin{gathered}
\mathrm{Y}_{1}=1,4810-0,0610\left(\frac{\varphi-65}{15}\right)+0,0647\left(\frac{t-20}{15}\right)-0,0400\left(\frac{V-0,2}{0,1}\right)++0,1560\left(\frac{t-20}{15}\right)^{2} \\
\mathrm{Y}_{2}=50,9792+3,100\left(\frac{\varphi-65}{15}\right)-2,676\left(\frac{t-20}{15}\right)-1,6971\left(\frac{V-0,2}{0,1}\right)-2,8711\left(\frac{\varphi-65}{15}\right)\left(\frac{t-20}{15}\right)- \\
\quad-1,3023\left(\frac{\varphi-65}{15}\right)^{2} \\
\mathrm{y}_{3}=43,0010-0,6352\left(\frac{\varphi-65}{15}\right)-0,6067\left(\frac{t-20}{15}\right)+0,2473\left(\frac{V-0,2}{0,1}\right)-0,5417\left(\frac{\varphi-65}{15}\right)^{2}- \\
-0,5700\left(\frac{t-20}{15}\right)^{2}-0,6701\left(\frac{V-0,2}{0,1}\right)^{2}
\end{gathered}
$$

for the cement packing shop:

$$
\begin{gathered}
\mathrm{Y} 1=17,3792-0,4503\left(\frac{V-0,2}{0,1}\right)+3,4042\left(\frac{t-20}{15}\right) \\
\mathrm{Y} 2=53,3390-0,4227\left(\frac{V-0,2}{0,1}\right)-0,8711\left(\frac{\varphi-65}{15}\right)\left(\frac{t-20}{15}\right)-0,8083\left(\frac{\varphi-65}{15}\right) 2- \\
-1,3023\left(\frac{t-20}{15}\right) * 2 \\
\mathrm{Y} 3=90,6042+4,1120\left(\frac{\varphi-65}{15}\right)-4,4631\left(\frac{t-20}{15}\right)-4,0010\left(\frac{V-0,2}{0,1}\right)-1,0421\left(\frac{V-0,2}{0,1}\right) * 2
\end{gathered}
$$

A mathematical model was developed to forecast the dust particle size distribution in the air of work areas at cement plants.

The particle size distribution of dust contained in the air of the work area can be expressed as the system $D(t)$ which at the time $t$ turns into any of the $n$ possible states $d_{1}, d_{2}$, $\ldots d_{n}$. Then, a random process proceeding in this system can be considered a Markovian process, i.e. for any point of time $t_{o}$ the probability of each of the states in the future $\left(t>t_{0}\right)$ depends solely on its state in the present and does not depend on its behavior in the past $\left(\mathrm{t}<\mathrm{t}_{0}\right)$.

The classification [5] enables us to identify the five groups of dust: 1 - very coarsedispersed, 2 - coarse-dispersed, 3 - medium-dispersed, 4 - fine-dispersed, 5 - very finedispersed dust.

The system $\mathrm{D}(\mathrm{t})$ can be expressed as a directed graph with its apices corresponding to the particle sizes which characterize the above groups of dust (fig.2). 


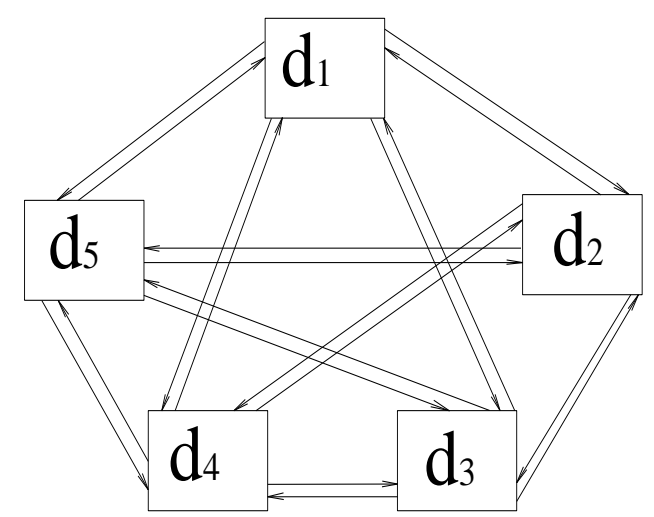

Fig. 2. Graph of dust particle size distribution in the work area

Once we denote the probability of the $\mathrm{i}$-th state at the time $\mathrm{t}$ (the probability of an event described as the system $D(t)$ being in the state $\left.d_{i}\right) p_{i}(t)=P\left\{D(t)=d_{i}\right\}$ at the time $t$, the sum of state probabilities for a system with discrete states at any point of time $t$ is equal to 1 , i.e.

$$
\sum_{\mathrm{i}=\mathrm{n}}^{5} \mathrm{p}_{\mathrm{i}}(\mathrm{t})=1
$$

Because at any given time the events $\left\{\mathrm{D}(\mathrm{t})=\mathrm{d}_{1}\right\},\left\{\mathrm{D}(\mathrm{t})=\mathrm{d}_{2}\right\}$, $\left\{\mathrm{D}(\mathrm{t})=\mathrm{d}_{3}\right\},\left\{\mathrm{D}(\mathrm{t})=\mathrm{d}_{4}\right\}$ form a complete group of incompatible events. Assuming that the transition from one state to another state is driven by the Poisson streams of events [6,7], the probability of transition of the system $D(t)$ from the state $d_{i}=D(t)$ to the state $d_{j}$ $=\mathrm{D}(\mathrm{t}+\Delta \mathrm{t} \quad)$ over an elementary time interval $\Delta \mathrm{t}$ is determined according to the formula:

$$
\mathrm{P}_{\mathrm{ij}}(\mathrm{t}) \approx \lambda_{\mathrm{ij}} \Delta \mathrm{t},
$$

where $\lambda_{\mathrm{ij}}$ - intensity of the Poisson stream of events making the system pass from the state $\mathrm{d}_{\mathrm{i}}$ to the state $\mathrm{d}_{\mathrm{j}}$

Accordingly, the graph can be described by a system of five ordinary Kolmogorov's differential equations:

$$
\frac{d p_{i}(t)}{d t}=\sum_{j=1}^{5} p_{j}(t) \lambda_{j i}-p_{i}(t) \sum_{j=1}^{5} \lambda_{i j}, i=1 \ldots 5
$$

This system is solved given the initial conditions

$$
\mathrm{p}_{1}(0)=\mathrm{p}_{1}{ }^{0}, \mathrm{p}_{2}(0)=\mathrm{p}_{2}{ }^{0}, \mathrm{p}_{3}(0)=\mathrm{p}_{3}{ }^{0}, \mathrm{p}_{4}(0)=\mathrm{p}_{4}{ }^{0}, \mathrm{p}_{5}(0)=\mathrm{p}_{5}{ }^{0} \text {. }
$$

The estimation of intensities $\lambda_{\mathrm{ij}}$ is based on the aggregate information presented in form of relative state frequencies of the system $\mathrm{D}(\mathrm{t})$ at each given time $\mathrm{t}$. As such, random data used for obtaining the particle size distribution must satisfy the stochastic equation

$$
\mathrm{y}_{\mathrm{j}}(\mathrm{t}+\Delta \mathrm{t})=\sum_{i=1}^{5} y_{i}(t) \lambda_{i j}+u_{j}(t+\Delta \mathrm{t})
$$

where $y_{i}(t)$ - vector of the observed frequencies of occurrence of the state $d_{i}$ (at the time $\left.t\right)$; $y_{j}(t+\Delta t)$ - vector of the observed frequencies of occurrence of the state $d_{j}$ (at the time $t+\Delta t$ ), and $u_{j}(t+\Delta t)$ - vector of casual errors [7].

The intensities $\lambda_{\mathrm{ij}}$ are estimated using the method of least squares, subject to certain restrictions, as regular least square estimates may not satisfy the conditions [6]: 


$$
\mathrm{o} \leq \lambda_{i j} \leq 1, \quad \mathrm{i}, \mathrm{j}=1, \ldots \ldots . .5 \text {. }
$$

In this case, the minimum of a quadratic form is to be determined as follows:

$$
\mathrm{F}=\sum_{J=1}^{5} u_{j}{ }^{\prime} u_{j}=\sum_{j=1}^{5}\left(y_{j}-X_{j} \lambda_{j}\right) \cdot\left(y_{j}-X_{j} \lambda_{j}\right)
$$

where $\mathrm{y}_{\mathrm{j}}=\left\{\mathrm{y}_{\mathrm{j}}(1), \mathrm{y}_{\mathrm{j}}(2), \ldots, \mathrm{y}_{\mathrm{j}}(\mathrm{T})\right\}$,

$$
\begin{gathered}
\mathrm{u}_{\mathrm{j}}=\left\{u_{j}(1), u_{j}(2), \ldots, u_{j}(T)\right\}, \lambda_{\mathrm{j}}=\left\{\lambda_{1 j}, \lambda_{2 j}, \ldots, \lambda_{5 j}\right\} \\
\mathrm{Xj}=\left\|\begin{array}{ccc}
y_{1}(0) & \ldots & y_{5}(0) \\
\ldots & \ldots & \ldots \\
y_{1}(T-1) & \ldots & y_{5}(T-1)
\end{array}\right\|
\end{gathered}
$$

where $\mathrm{T}$ - length of the sample.

The root-mean-square error of approximating intensities is determined according to the formula

$$
\varepsilon_{j}=\sqrt{\sum_{t=1}^{T} u_{j}^{2}(t) / T},
$$

where $\mathrm{u}_{j}(\mathrm{t})$ - components of the vector of casual errors.

To estimate the intensities of transition probability, we will use the methodology [7]. For this, let us consider the example of the cement milling shop. The equipment layout diagram indicating the points of measurements is given in fig. 3. The measurements were taken according to the methodology [8].

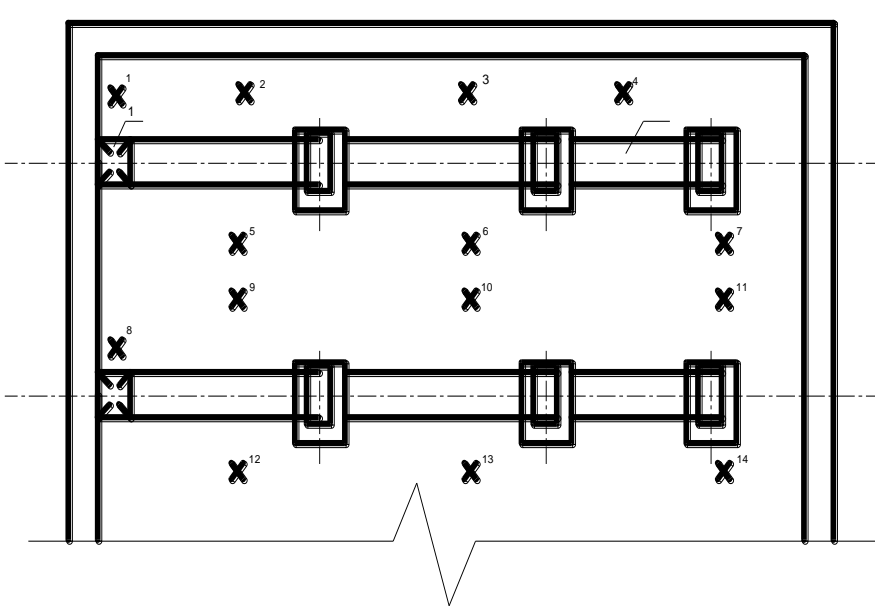

Fig. 3. Layout of measurement points in the work area of the milling shop

Samples were taken at 14 points (fig. 4) once per shift during one month. A total of 30 measurement series were obtained. The number of measurements was assumed so as to provide a homogeneous sample. During the measuring process, the microclimate parameters conformed to the rated levels. 
By way of an experimental study, samples were obtained simulating the variation of the shift-averaged median diameter $\mathrm{d}_{\mathrm{m}}$. Overall, 30 implementations of a random variation process for the parameter $\mathrm{d}_{\mathrm{m}}$ were built, one of which is shown in fig. 4 .

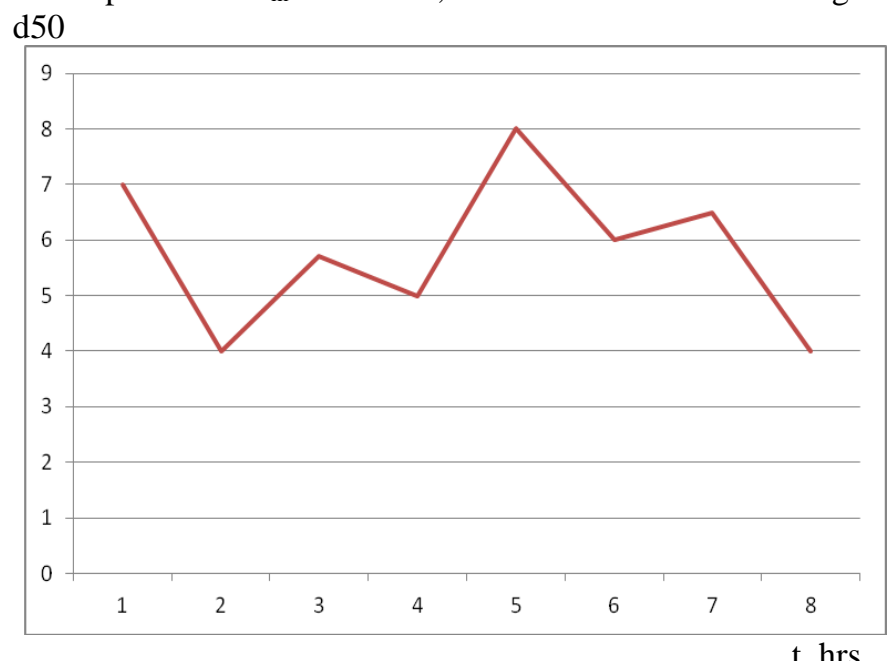

Fig. 4. Model of implementing a random variation process for the parameter $\mathrm{d}_{\mathrm{m}}$

As a result, a matrix of intensities was obtained for transition probabilities.

$$
\left\|\lambda_{i j}\right\|=\left\|\begin{array}{|ccccc}
0,327 & 0,134 & 0,288 & 0 & 0 \\
0 & 0 & 0 & 1,0 & 0 \\
0 & 0 & 0,274 & 0,415 & 0,163 \\
0,013 & 0,019 & 0,334 & 0,405 & 0,122 \\
0 & 0 & 0,714 & 0,122 & 0
\end{array}\right\|
$$

By placing the value of the matrix elements (16) in the equation (8) we obtained a system of ordinary differential equations to be solved with the $4^{\text {th }}$ order Runge-Kutta method given the initial conditions (9). The probability of work area contamination with dust having the corresponding particle side distribution is equal to: $\mathrm{p}_{1}=0.018, \mathrm{p}_{2}=0.024, \mathrm{p}_{3}=0.438, \mathrm{p}_{4}=0.405$, $\mathrm{p}_{5}=0.115$.

The root-mean-square error of approximating the intensities $\lambda_{\mathrm{ij}}$ is as follows: $\varepsilon_{1}=0.02$, $\varepsilon_{2}=0.03, \varepsilon_{3}=0.25, \varepsilon_{4}=0.2, \varepsilon_{5}=0.11$.

The most probable system state is the presence of medium-dispersed and fine-dispersed dust, while the probability of very fine-dispersed, coarse- and very coarse-dispersed dust states is low.

\section{Conclusion}

1. The particle size distribution of dust in the air of the work area has been determined for the cement milling shops, mill feeder, rotary kiln operator's work area and the cement packing shop in the packing machine operator's work area.

2. An experimental study has been conducted to explore the effects of microclimate parameters (humidity, temperature, mobility of the air in work area) on the dust particle size distribution of in cement milling shop and cement packing shop. Regression equations have been obtained, which help link and explore the influence of variable 
factors on the optimization parameters (the content of $10 \mu \mathrm{m}$ (PM10) particles, $2.5 \mu \mathrm{m}$ particles $\mathrm{D}(\mathrm{PM} 2.5)$, and median-size particles $\mathrm{D}(50)$ in the air of the work area).

3. A mathematical model has been developed to forecast the dust particle size distribution in the air of the work area at cement plants. A conclusion has been made that the most probable option is the presence of medium-dispersed and fine-dispersed dust in the air of the work area, while the probability of very fine-dispersed, coarse- and very coarsedispersed dust states is low.

\section{References}

1. V.N. Azardov, N.V. Menzelintseva, N.Yu. Karapuzova, E.O. Fomina, N.A. Pshenichkina Experimental estimation of dust factor at cement plants, V.N. Azardov, Bull. of VGASU, 2016, Architecture and Construction series, 44 (63), 134-145.

2. V.N Azarov, N.V. Menzelintseva, N.U. Karapuzova, A. Redhwan, Main Trends of Dust Conditions Normalizing at Cement Plants, International Review of Civil Engineering, 6 (6), 145-150 (2015).

3. N.V. Menzelintseva, N.Yu. Karapuzova, E.O. Fomina, S.I. Stefanenko, Experimental Study of Efficiency of Cement Dust Collection by Aspiration Systems, Bull. of VGASU, Architecture and Construction series, 25 (44), 249-252 (2011).

4. S.L. Akhnazarova, V.V. Kafarov, Methods for Experiment Optimization in Chemistry and Chemical Technology (Higher School, Moscow, 1985).

5. A.I. Pirumov, Dedusting of Air (Stroizdat, Moscow, 1981).

6. V.N. Azarov, N.V. Menzelintseva, N.Yu. Karapuzova, E.O. Fomina, N.A. Pshenichkina, Mathematical Model to Forecast Dust Particle Size Distribution in the Air of Work Areas at Cement Plants in Assessing the Dust Situation, Bull. of VGASU, Architecture and Construction series, 44 (63), 134-145 (2016).

7. T.V. Gavrilenko, O.V. Adamayev, Assessing Environmental Safety of Atmospheric Air by Solving Kolmogorov's Equations, Bull. of the Kemerovo State University, 2 (4) (52), 37-43 (2012).

8. V.N. Azarov, Methodology for Identifying Intensities of Dust Emission by Process Equipment, Volgograd, 2002, p. 8, VINITI Dept., 15.07.2002, No. 1332 